\title{
Pancoast Tumor Masquerading as Cervical Spondylosis : a Case Report
}

\author{
Gupta MK, ${ }^{* *}$ Dhungel K, ${ }^{1}$ Ahmad $K,{ }^{1}$ Rauniyar RK, ${ }^{1}$ Ansari $S^{1}$ \\ ${ }^{1}$ Department of Radiodiagnosis and Imaging, B. P. Koirala Institute of Health Sciences, Dharan, Nepal.
}

\author{
* Corresponding Author: \\ Dr. Mukesh Kumar Gupta, \\ Associate Professor, \\ Department of Radiodiagnosis \& Imaging, \\ B.P. Koirala Institute of Health Sciences, Dharan, Nepal. \\ Phone: +977-9842025813, \\ Email: mukeshgupta148@yahoo.com

\section{Citation} \\ Gupta MK, Dhungel K, Ahmad K, Rauniyar RK, Ansari S. \\ Pancoast Tumor Masquerading as Cervical Spondylosis: \\ a Case Report. Nepal Journal of Medical Sciences \\ 2013;2(2):184-6.
}

\begin{abstract}
Pancoast tumor is a primary carcinoma occurring at the apex of the lungs. These tumors have distinct clinical presentation because of their anatomical location and the resultant clinical entity, called Pancoast syndrome, presents as severe pain in the neck, shoulder and arm and as Horner's syndrome. The initial symptoms are most frequently musculoskeletal in nature and the pulmonary symptoms of lung cancer are rather rare resulting in delayed diagnosis of this condition in majority of the cases. We report a case of 65-year-old female who presented with pain in the neck, left shoulder and arm along with numbness and tingling sensation in her forearm and hand mimicking clinically as cervical spondylosis. On detailed evaluation, she was diagnosed to have Pancoast tumor. This case report highlights the clinical presentation of Pancoast tumor and emphasizes the importance of its inclusion in the differential diagnosis of patients presenting with persistent pain in the neck and shoulder.
\end{abstract}

Keywords: Cervical spondylosis; lung carcinoma; pancoast tumor; pancoast syndrome; superior sulcus tumor

\section{Introduction:}

The Pancoast tumour is a primary malignant tumor occurring at the apex of the lung in the area of the sulcus formed by passage of the subclavian artery which can invade the adjacent ribs, the vertebral bodies, the brachial plexus, the subclavian vessels and the stellate ganglion. ${ }^{1-3}$ Pancoast syndrome is characterized by pain, which may arise in the shoulder or chest wall and may radiate to the neck. Horner's syndrome, which consists of ptosis, meiosis and anhydrosis, results from invasion of the paravertebral sympathetic chain. Weakness and atrophy of the hand and parasthesias are common clinical findings resulting from invasion of the $\mathrm{C} 8$ and $\mathrm{T} 1$ nerve roots of the brachial plexus. More infrequent manifestations include supraclavicular adenopathy, superior vena cava syndrome and involvement of the phrenic or laryngeal nerves. ${ }^{4}$ The aim of this case report is to emphasize the importance of high index of clinical suspicion of Pancoast tumor in patients presenting with persistent pain in the neck and shoulder. The clinical presentation, radiograph and CT findings of the patient with Pancoast tumor are presented here.

\section{Case report:}

A 65-year-old female with history of smoking, presented with the complaints of progressive pain in neck, left shoulder and arm to a local physician who diagnosed her as a case of cervical spondylosis based on the clinical findings and put on conservative management. During the course of treatment, her pain became severe and excruciating with numbness and tingling sensation in the forearm and hand. Then, the patient was referred to B.P. Koirala Institute of Health Sciences, 
Dharan, Nepal, which is a tertiary care center, for further evaluation. Chest radiograph showed a homogenous opacity in the left upper zone in the apical region with destruction of adjacent ribs [Figure1].

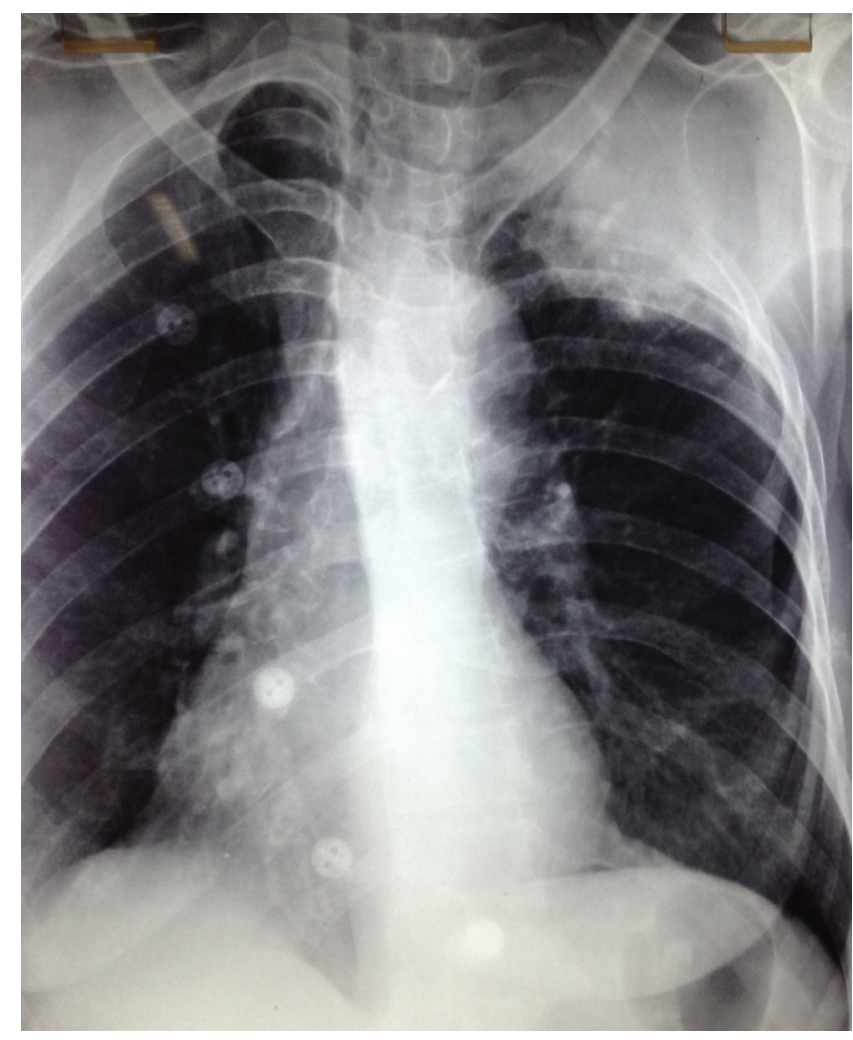

Figure 1: PA chest radiograph shows a homogenous opacity in left upper zone in the apical region associated with destruction of the adjacent ribs.

Multi-detector Computed Tomography (MDCT) of thorax in axial and reformatted coronal planes [Figure 2, 3 and 4] revealed an irregular, lobulated, homogeneous enhancing mass of size $7 \times 5 \times 4.5 \mathrm{~cm}$ with few tiny calcific foci within in left apical region.

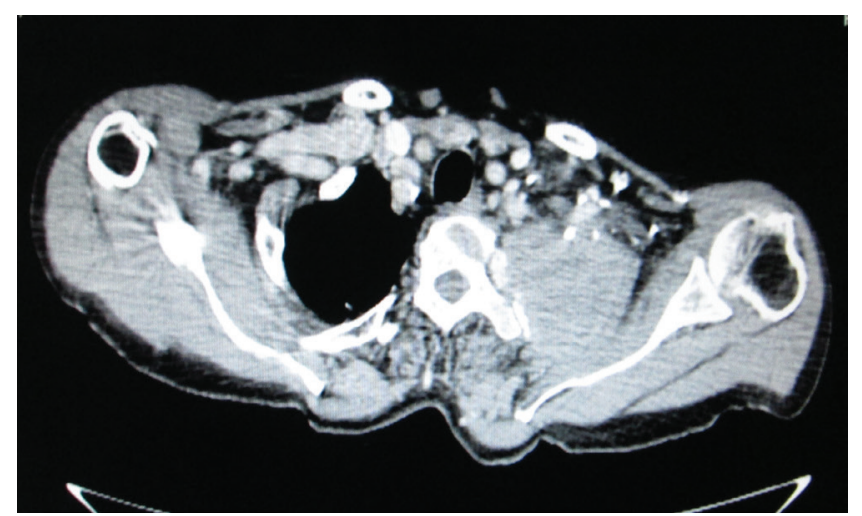

Figure 2: Contrast enhanced axial CT image shows a homogeneous enhancing lung mass in left apical region causing destruction of the adjacent ribs.

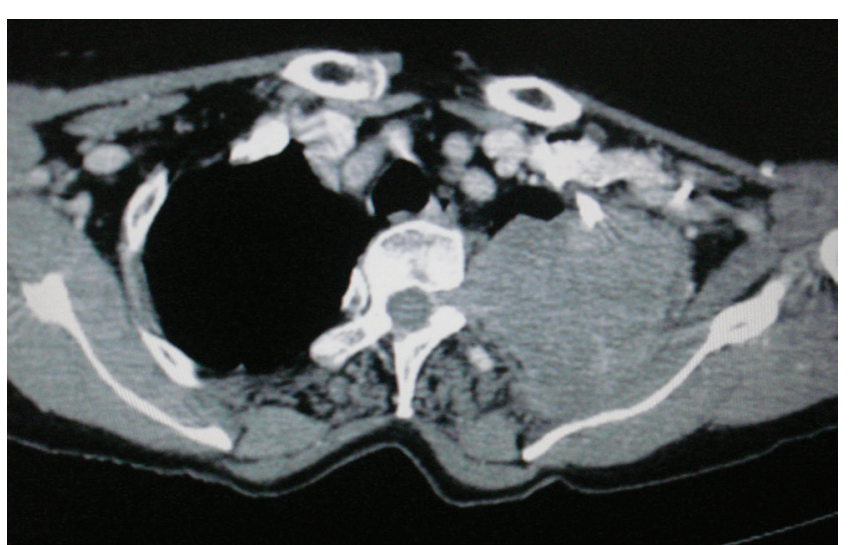

Figure 3: Axial CT image shows the apical tumor invading the left neural foramen.

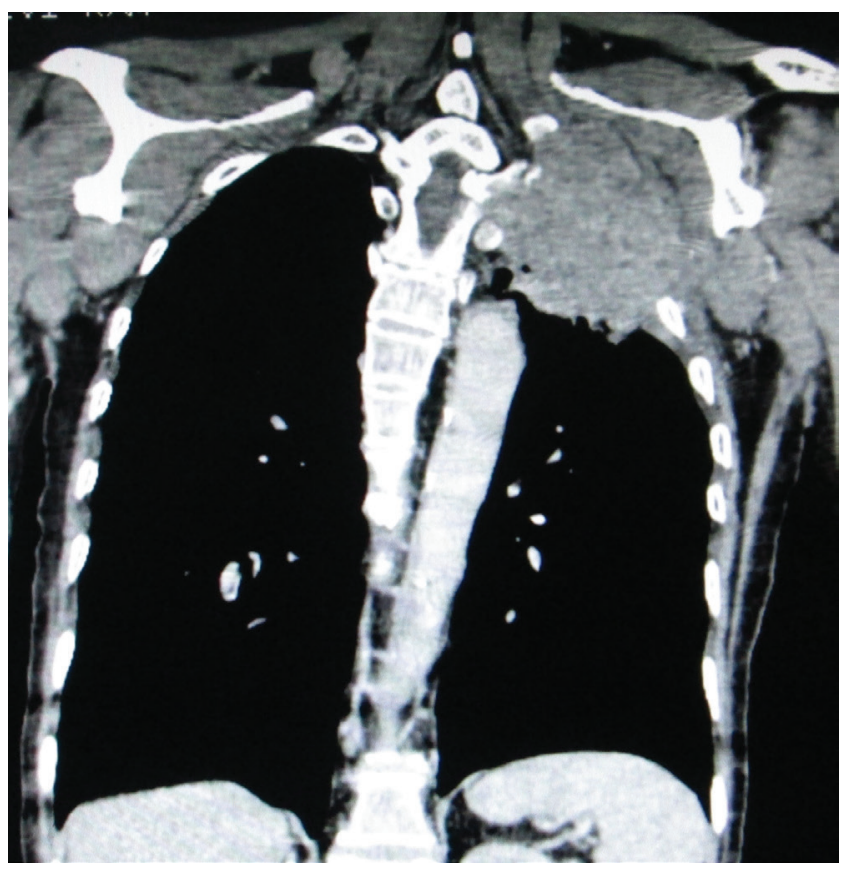

Figure 4: Coronal reformatted CT image shows the left apical tumor invading the chest wall and paravertebral soft tissues with destruction of the ribs and transverse process of the vertebra.

The mass was infiltrating the postero-lateral chest wall and causing destruction of the posterior part of $1^{\text {st }}, 2^{\text {nd }}, 3^{\text {rd }}$ and $4^{\text {th }}$ ribs. The mass was also causing erosion of the transverse process of $\mathrm{T} 2$ vertebra and invading the neural foramen at T1-T2 level on left side. A needle aspiration from the mass revealed the diagnosis of squamous cell carcinoma and the patient was referred to Cancer Center for further management.

\section{Discussion:}

Pancoast tumour is an infrequent subtype of primary lung malignancy which accounts for approximately $2-5 \%$ of all the lung cancers. The peripheral location of the tumor in the 
apex of the lung generates clinical symptoms that may easily be attributed to other causes, like musculoskeletal disorders. The initial symptoms associated with Pancoast tumour are most frequently musculoskeletal in nature. In fact, more than $90 \%$ of patients initially presents with shoulder pain, rather than with pulmonary symptoms because the extra pulmonary bulk of the tumor mass can invade the thoracic inlet and affect the adjacent structures such as spinal nerve roots, subclavian vessels, sympathetic chain and its stellate ganglion., ${ }^{2,5-7}$ In our case also pulmonary symptoms of malignancy such as cough, hemoptysis and dyspnea were absent. This case initially presented with pain in the neck, shoulder and arm with development of tingling sensation and numbness in the forearm and hand later in the course of disease. Deng et $\mathrm{al}^{8}$ in a study reported misdiagnosis of Pancoast tumour as musculoskeletal disorder and pulmonary tuberculosis in $73 \%$ of cases.

Early detection of Pancoast tumor may be made by careful inspection of radiographic investigation of the upper lung field in apical lordotic, full size PA and lateral views which can reveal a small homogeneous shadow in the apical region of the lung, with the occasional evidence of erosion and destruction of adjacent ribs and vertebrae. A small soft tissue mass in the apical region may not be seen on radiograph due to superimposition of the bone shadow at this level. When there is high index of clinical suspicion, other imaging modalities such as CT scan or MRI may be required to exclude a Pancoast tumor even when the plain radiograph changes are subtle., ${ }^{2,9}$ Bone involvement is best shown by CT scan, but MRI is excellent in evaluation of soft tissue involvement, and is far more sensitive (88\%) and specific $(100 \%)$ for local staging of the tumor. The role of ultrasound is limited, however it may be useful in aiding percutaneous biopsy as it is able to visualize the external component of the tumor via an intercostal or supraclavicular acoustic window. ${ }^{10,11}$ Biopsy via bronchoscopy has a low yield due to the anatomical location of the tumor mass. The diagnostic procedure of choice for material acquisition is image-guided needle aspiration biopsy, which has low complication rates. ${ }^{4,12,13}$

\section{Conclusion:}

As Pancoasttumors are frequently misdiagnosed as variety of musculoskeletal disorders, it is important to havehigh index of clinical suspicion of this entity in patients presenting with persistent pain in the neck and shoulder, particularly if the patient is smoker, so that early diagnosis can be made which is crucial for the management.

\section{Conflicts of interest: None}

\section{References:}

1. Paulson DL. Carcinomas in the superior pulmonary sulcus. J ThoracCardiovasc Surg 1975;70:1095-102.

2. Spengler DM, Kirsh MM, Kaufer H. Orthopaedic aspects and early diagnosis of superior sulcus lung tumour (Pancoast). J Bone Joint Surg 1973;55:1645-50.

3. Teixeira JP. Concerning the Pancoast tumor: what is the superior pulmonary sulcus? Ann Thorac Surg $1983 ; 35: 577-8$.

4. Arcasoy SM, Jett JR. Superior pulmonary sulcus tumors and Pancoast's syndrome. N Engl J Med 1997;337:1370-6.

5. Kovach SG, Huslig EL. Shoulder pain and Pancoast tumor: a diagnostic dilemma. J Manipulative PhysiolTher $1984 ; 7: 25-31$.

6. Sagerup CM, Brustugun OT, Jørgensen L. A 67-year old man with right arm paresthesias. Tidsskr Nor Laegeforen 2009;129:2613-5.

7. Pancoast HK. Superior pulmonary sulcus tumors: tumor characterized by pain, Homer's syndrome, destruction of bone, and atrophy of hand muscles. JAMA 1932;99:1391-6.

8. Deng PB, Luo YY, Hu CP, et al. Misdiagnosis of pancoast cancer: analysis of 26 cases. Zhonghua Jie He He Hu Xi ZaZhi 2011;34:663-5.

9. Brown C. Compressive, invasive referred pain in the shoulder. Clin Orthop 1983;173:55-62.

10. Yang PC, Lee LN, Luh KT, et al. Ultrasonography of Pancoast tumor. Chest 1988;94: 124-8.

11. Lung cancer, principles and practice. Lippincott Williams and Wilkins. (2005).

12. Archie VC, Thomas CR Jr. Superior sulcus tumors: a mini-review. Oncologist 2004;9:550-5.

13. Khosravi SP. Pancoast's syndrome (superior pulmonary sulcus tumor): review of the literature [Article in Spanish]. An Med Interna 2005;22:194-6. 